\title{
Radio Frequency Selective Addressing of Localized Particles in a Periodic Potential
}

\author{
H. Ott, E. de Mirandes, F. Ferlaino, G. Roati, V. Türck, G. Modugno, and M. Inguscio \\ LENS and Dipartimento di Fisica, Università di Firenze, \\ and INFM, Via Nello Carrara 1, 50019 Sesto Fiorentino, Italy
}

(Dated: October 30, 2018)

\begin{abstract}
We study the localization and addressability of ultra cold atoms in a combined parabolic and periodic potential. Such a potential supports the existence of localized stationary states and we show that using a radio frequency field allows to selectively address the atoms in these states. This method is used to measure the energy and momentum distribution of the atoms in the localized states. We also discuss possible extensions of this scheme to address and manipulate particles in single lattice sites.
\end{abstract}

PACS numbers: 03.65.-w, 03.75.Lm, 03.65.Ge, 03.67.-a

Periodic potentials have been used with great success in a series of experiments with ultra cold atoms [1, 2, 3, 4]. For a trapped sample the periodic potential is usually accompanied with an additional parabolic confinement [3, 4]. In most experiments involving trapped Bose-Einstein condensates only the properties of the ground state, which are not changed dramatically by the parabolic confinement, are important. However, for the excited states of the system the additional harmonic confinement must be taken into account. This is especially true for atomic Fermi gases, where the Pauli principle enforces a population of higher energetic states. The qualitative different nature of the single particle states can be seen from recent experiments with ultra cold fermions which have found evidence for a localization of the particles in such a potential [5, 6, 7]. In contrast to the Mott insulating phase [4], this localization process is independent from the interaction and is a pure consequence of the potential shape. It is therefore important to understand its properties and physical consequences, nonetheless because most of the theoretical work on quantum phase transitions involving fermions 8, 9, 10, 11 are based on pure homogeneous systems. A combined periodic and parabolic potential is also interesting for possible applications in quantum information and was recently proposed for the implementation of a qubit register for fermions [12. A possible addressability of individual atoms in single lattice sites is thereby an intriguing vision.

In this work we study ultra cold atoms in a parabolic magnetic potential which is superimposed in its weak direction with a one dimensional optical lattice. The combined potential possesses two distinct classes of eigenstates, which - depending on their energy - either extend symmetrically around the trap center or are localized on the sides of the potential. We use a radio frequency technique to address the particles in localized states and to measure the density of state along the lattice direction as well as the momentum distribution of the particles. Due to the localization of the particles, the radio frequency field addresses the particles in a defined spatial region and we discuss the possibility of extend- ing this scheme to manipulate individual atoms in single lattice sites.

In the experiment we prepare an ultra cold cloud of ${ }^{87} \mathrm{Rb}$ in the combined potential by forced evaporative cooling. The magnetic trapping potential has an axial and radial oscillation frequency of $\omega_{\mathrm{a}}=2 \pi \times 16 \mathrm{~Hz}$ and $\omega_{\mathrm{r}}=2 \pi \times 197 \mathrm{~Hz}$ and the optical lattice $(\lambda=830 \mathrm{~nm})$ can be adjusted between $0<s<10$, where $s$ measures the potential height in units of the recoil energy $E_{r}=h^{2} / 2 m \lambda^{2}$. The atoms are prepared in the spinpolarized $\left|F=2, m_{\mathrm{F}}=2\right\rangle$ state and the temperature is between 500 and $600 \mathrm{nK}$. Here we are only interested in the single particle behavior; in this frame bosons and fermions show the same features, as long as interparticle interactions can be neglected.

To understand the properties of the combined potential we first solve the 1D Schrödinger equation in the direction of the lattice,

$\left[-\frac{\hbar^{2}}{2 m} \frac{\partial^{2}}{\partial x^{2}}+\frac{1}{2} m \omega^{2} x^{2}+\frac{s}{2} E_{r}(1-\cos 4 \pi x / \lambda)\right] \psi=E_{n} \psi$,

where $E_{n}$ is the energy of the $n$th eigenstate. This Hamiltonian has also recently been studied in tight binding approximation 13, 14]. In Fig. 1a we show a density plot of the first 1000 eigenfunctions of (11). Each line in Fig. 17 corresponds to a density plot of the wave function. For low energies we find delocalized states that spread symmetrically around the potential minimum. Above a threshold energy, the wave functions of the eigenstates become localized on both sides of the potential. If we look at higher energies, a second group of eigenstates appears, centered again around the trap minimum. It is straightforward to identify this shell-like structure with the well known band picture for a pure periodic potential. This becomes particularly clear if one looks at the accessible energy values for a given position which correspond to the calculated bandwidth $E_{\mathrm{bw}}$ and band gap $E_{\text {gap }}$ of a pure sinusoidal potential, only shifted in energy by the local value of the parabolic potential. As a direct consequence, the system does not have an absolute but a spatially varying energy gap. The temperature of the 


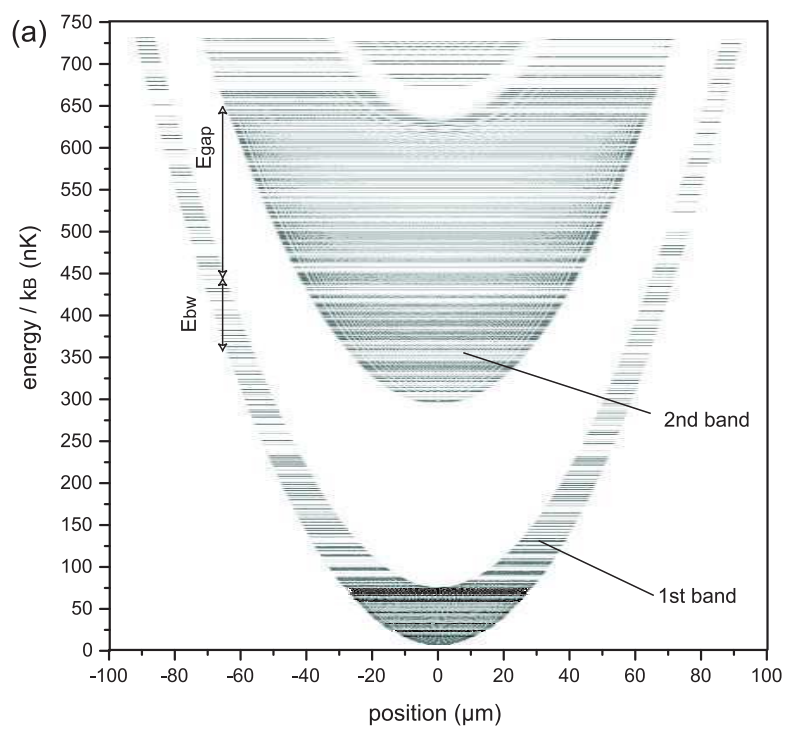

(b)

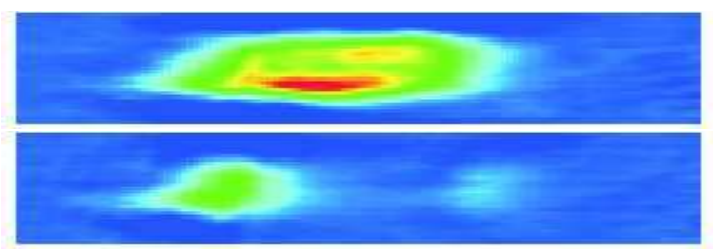

FIG. 1: (a) Spectrum of the Hamiltonian: representation of the $1 \mathrm{D}$ spectrum of the single particle Schrödinger equation for a combined parabolic and periodic potential with $s=3$. Each line represents one eigenstate of the system, which is plotted as density profile in grayscale. The vertical position of the profile corresponds to the energy of the eigenstate. (b) Cloud of atoms without rf field (upper image) and with rf field (lower image) after $1.5 \mathrm{~ms}$ time of flight.

cloud is chosen that the bandwidth of the first band is much smaller than the average energy of the atoms, thus providing a high population of the localized states. In the two radial directions, the particles occupy the harmonic oscillator states.

We now introduce our experimental technique to prepare and address the particles in the localized states. After the end of the evaporation a radio frequency (rf) field is applied in order to induce spin flip transitions. Because of the magnetic radial confinement this removes all atoms whose wavefunction has a spatial overlap with the magnetic field shell where the resonance condition $h \nu=$ $\mu_{\mathrm{B}} B(\mathbf{r}) / 2$ is fulfilled. Periodically sweeping ( $1 \mathrm{kHz}$ rate) the radio frequency within an interval $\Delta \nu=\nu_{\mathrm{up}}-\nu_{\text {low }}$ we define a spatial region in which the atoms are removed from the potential. After $100 \mathrm{~ms}$ of rf field we image the atoms which are left in the potential. In Fig.10 we show an absorption image of the atomic cloud after $1.5 \mathrm{~ms}$ time of flight without and with rf field. In the latter case we remain with two clouds, located at the edges of the original cloud. Atoms in these two clouds are trapped in localized states and the result directly shows, that the rf field is (a)

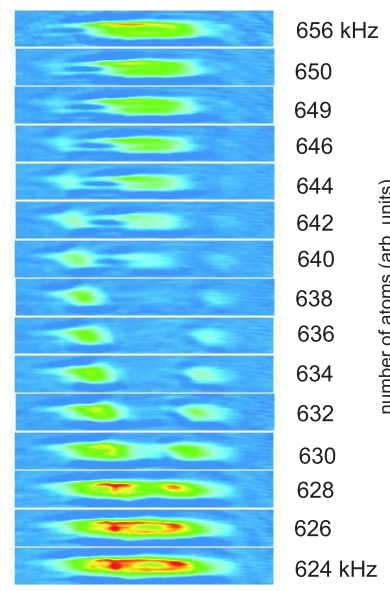

(b)

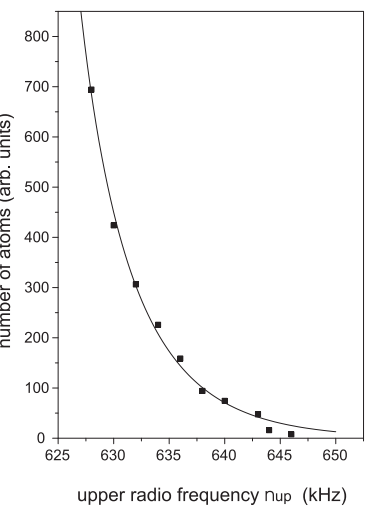

FIG. 2: Energy distribution of localized states in a lattice with $s=9$. (a) Scan of the rf field through the cloud. The indicated frequencies are the upper frequency $\nu_{\mathrm{up}}$ of the $\mathrm{rf}$ field, the sweep amplitude of the field is $3 \mathrm{kHz}$. (b) Atom number in the left cloud in dependence on $\nu_{\text {up }}$. The straight line is a fit with an axial density of states that is proportional to $E^{-1 / 2}$ (see text).

capable to address the atoms in a defined spatial region. We have checked that (i) without the optical lattice all atoms are removed from the trapping potential and (ii) after switching off the rf field the atoms remain on their position in the trap. Only for small lattice heights we can observe a slow motion of the two clouds towards the trap center. This is due to presence of collisions which allow the bosons to hop between different localized states [6]. For a spin-polarized Fermi gas this effect is absent.

In our recent work [5, 6, 7] we were able to detect the localization of the atoms by looking at the center of mass position of the whole cloud. Here, we can employ this kind of rf spectroscopy to look at the energy distribution of the atoms in the localized states. In Fig.20 we show a series of absorption images where we have scanned the rf field fixing the frequency interval $\Delta \nu=3 \mathrm{kHz}$. Increasing the $\mathrm{rf}$ frequency we start to remove atoms from the center of the trap. The hole in the spatial distribution deepens, until the lower frequency bound is higher than the resonance frequency at the trap bottom: atoms in the center are no longer removed from the potential and we observe three clouds until for even higher frequencies the displaced peaks disappear and the cloud is again unaffected by the rf field. In Fig.2r we show the number of atoms in the left cloud in dependence on upper frequency of the rf field. Due to the localization these atoms have an axial energy which is higher than $E_{\mathrm{up}}=2 h\left(\nu_{\mathrm{up}-\nu_{0}}\right)$, where $\nu_{0}$ is the resonance frequency in the trap center. Thus, the number of atoms in the cloud is determined by the density of states in the axial direction: $N \propto \int_{E_{\mathrm{u} p}}^{\infty} n\left(E_{\mathrm{a}}\right) \rho_{\mathrm{a}}\left(E_{\mathrm{a}}\right) d E_{\mathrm{a}}$, where $\rho_{\mathrm{a}}\left(E_{\mathrm{a}}\right)$ is the density of states in the axial direction and $n\left(E_{\mathrm{a}}\right)=e^{-E_{\mathrm{a}} / k_{\mathrm{B}} T}$ is the axial energy distribution. As 


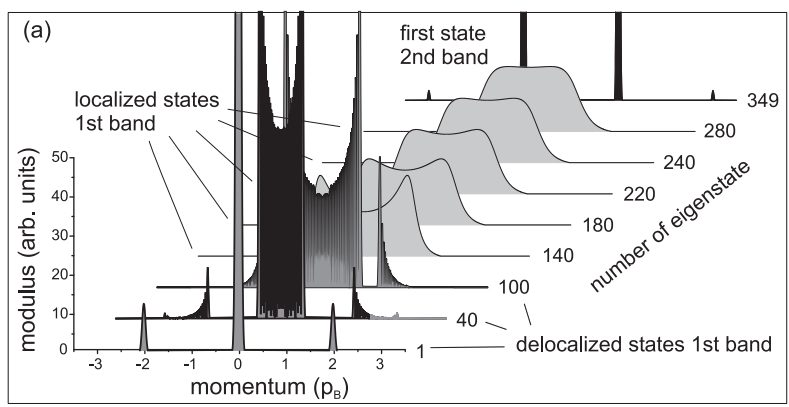

(b)
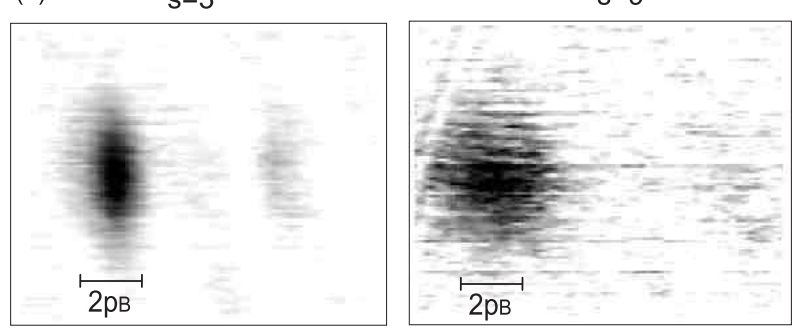

FIG. 3: Momentum distribution of the localized states. (a) Fourier transform of selected eigenstates of the spectrum shown in Fig.11. The first three states are delocalized and lie within the first band, the last state is the lowest state of the second band. The states in between are localized states. (b) Absorption image of a cloud of atoms such as shown in Fig.20 after $10 \mathrm{~ms}$ time of flight for $s=3$ and $s=9$.

shown in Ref. 14] the density of states for energies larger than the bandwidth of the first band is predicted to be proportional to $E^{-1 / 2}$. Using this value we fit our data leaving the temperature and a constant of proportionality as free parameters [15]. The result which is shown in Fig.20 is consistent with our data.

We now turn to the momentum distribution of the localized states. For a potential depth of $s=3$ we have calculated the Fourier transform of the wave functions of the eigenstates. Fig. 3 shows the momentum distribution for selected eigenstates within the first and second band. The lowest eigenstate shows the well known peak distribution at multiples of twice the Bragg momentum. With increasing energy these peaks broaden and develop a substructure. The states of our interest are the localized states, whose distribution spreads over the first Brillouin zone $\left( \pm p_{\mathrm{B}}\right)$ and is similar for all states, regardless the energy of the state. In the radial direction, the momentum distribution is determined by the temperature of the cloud. Consequently, localized clouds as shown in Fig. 2 are expected to exhibit an anisotropic expansion. In Fig. 3b we show an absorption image of a localized cloud after $10 \mathrm{~ms}$ time of flight for $s=3$ and $s=9$. The measured aspect ratio of 2.5 for $s=3$ is highly anisotropic and directly proves the nonclassical momentum distribution of the localized states. For $s=9$ the cloud expands much faster in the direction along the lattice (horizontal direction). Indeed we calculate a 2 times (a)
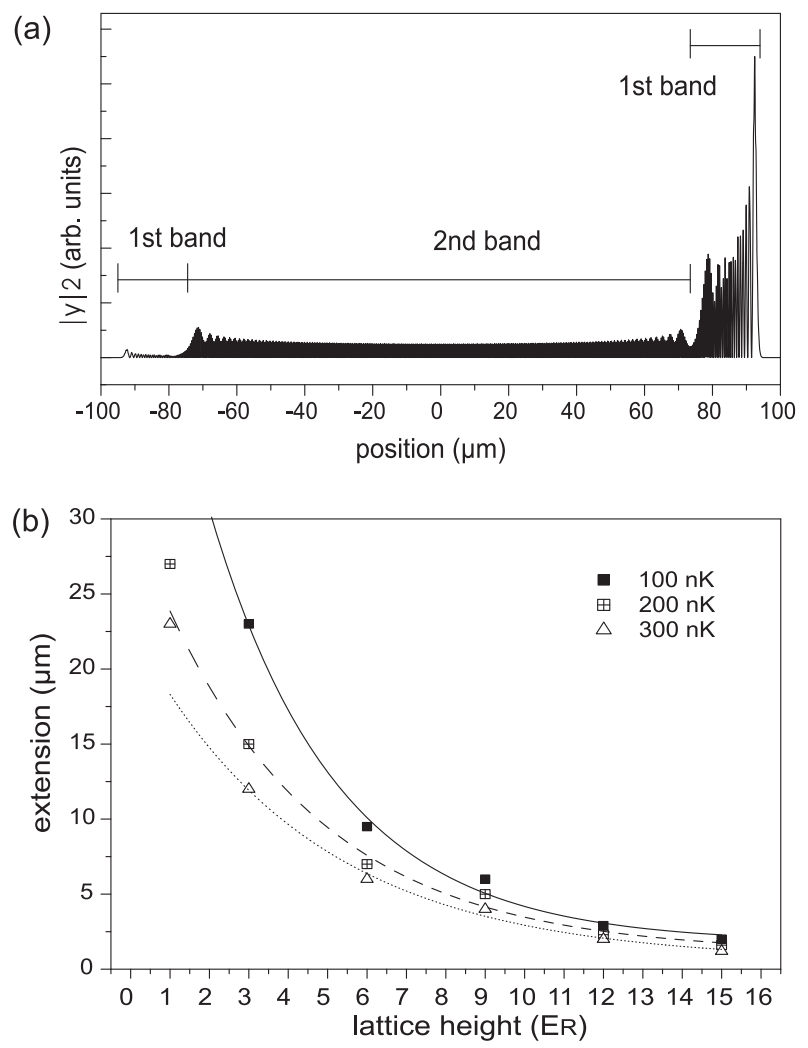

FIG. 4: (a) Tunnelling between the bands: density distribution of the 594th eigenstate for a potential with $s=0.3$. (b) Extension of the localized states in the first band in dependence on the lattice height for three different energies $\left(k_{\mathrm{B}} \times 100\right.$ $\mathrm{nK}, k_{\mathrm{B}} \times 200 \mathrm{nK}$, and $k_{\mathrm{B}} \times 300 \mathrm{nK}$, .

larger momentum distribution for $s=9$ with respect to $s=3$ which leads to a nearly isotropic expansion.

We finish this work with discussing the localization process and its possible applications. For small lattice heights, the localization of the states is prevented by inter band transitions and as an example we show in Fig. [4. an excited state for $s=0.3$. The wave function exhibits substantial contributions from both bands and a particle in this state is no longer localized. On the other side the extension of the localized state shrinks with increasing lattice height (Fig.4b) and the smallest possible extension is given by the ground state in each lattice site. For our parameters we find that for $s=30$ the eigenstates are mainly located within a single lattice site. This result is of particular interest because it shows that for similar experimental conditions like in the Mott insulator experiment [4] a localization of the particles within one lattice site is possible without a repulsive interaction. Indeed, Viverit et al. 12] have shown that if an atomic Fermi gas is loaded in a combined parabolic and periodic potential even an occupancy with exactly one atom per lattice site can be achieved. Another intriguing consequence of the localization is the addressability of single 
lattice sites: the potential gradient discriminates the resonance condition for an atomic transition in each lattice site if the transition depends on the external potential. In our setup the magnetic potential leads to a spatially varying Zeeman splitting within the $|F=2\rangle$-manyfold and thus a very weak radio frequency should allow - in principle - for the manipulation of the atoms within one lattice site. To get a reasonable discrimination and a sufficiently high Rabi frequency, the resonance condition between adjacent lattice sites should be shifted by something about $10 \mathrm{kHz}$ which would require a gradient of $300 \mathrm{G} / \mathrm{cm}$ [18]. Thereby a linear potential is more favorable than a parabolic one where the frequency shift is changing along the lattice. For well defined experimental conditions it would be also desirable to have an optical confinement in the radial direction because otherwise particles with lower axial but higher radial energy might also be resonant with the radio frequency.

In conclusion we have proved that particles in a combined periodic and parabolic potential are trapped in localized states. We have used a radio frequency field to induce spatially resolved transitions in order to remove the particles from the potential. Doing so it was possible to measure the axial density of states and the momentum distribution of the localized states. The experiment has implications in various directions. First it shows that an inhomogeneous periodic potential exhibit a qualitatively different phenomenology compared to a homogeneous system. Second the experiment directly evidences a new localization mechanism which is independent from the interaction. Especially for atomic Fermi gases such a potential can be interesting to create single lattice occupancy [12]. Third we show that in principle the particles can be addressed spatially resolved. For future experiments it should be possible to extend the scheme to the manipulation of particles in single lattice sites which would constitute a major progress in "quantum engineering" with ultra cold atoms.

This work was supported by MIUR, by EU under Contract. No. HPRICT1999-00111, and by INFM, PRA "Photonmatter". H. O. and V.T. were supported by EU with Marie Curie Individual Fellowships, under Contracts No. HPMF-CT-2002-01958 and HPMF-CT-200201649 .
[1] M. Ben Dahan, E. Peik, J. Reichel, Y. Castin, and C. Salomon, Phys. Rev. Lett. 76, 4508 (1996).

[2] S. R. Wilkinson, C. F. Bharucha, K. W. Madison, Qian Niu, and M. G. Raizen, Phys. Rev. Lett. 76, 4512 (1996).

[3] F. S. Cataliotti, S. Burger, C. Fort, P. Maddaloni, F. Minardi, A. Trombettoni, A. Smerzi, and M. Inguscio, Science 293, 843 (2001).

[4] M. Greiner, O. Mandel, T. Esslinger, T.W. Hänsch, and I. Bloch, Nature 415, 39 (2002).

[5] G. Modugno, F. Ferlaino, R. Heidemann, G. Roati, and M. Inguscio, Phys. Rev. A 68, 011601(R) (2003).

[6] H. Ott, E. de Mirandes, F. Ferlaino, G. Roati, G. Modugno, and M. Inguscio, cond-mat/0311261

[7] L. Pezzé, L. Pitaevskii, A. Smerzi, S. Stringari, G. Modugno, E. de Mirandes, F. Ferlaino, H. Ott, G. Roati, and M. Inguscio, cond-mat/0401643

[8] M. Rigol, A. Muramatsu, G. G. Batrouni, and R. T. Scalettar, Phys. Rev. Lett. 91, 130403 (2003).

[9] A. Albus, F. Illuminati, and J. Eisert, Phys. Rev. A 68, 023606 (2003).

[10] H. P. Büchler and G. Blatter, Phys. Rev. Lett. 91, 130404 (2003).

[11] M. Lewenstein, L. Santos, M. A. Baranov, H. Fehrmann, Phys. Rev. Lett. 92050401 (2004).

[12] L. Viverit, C. Menotti, T. Calarco, and A. Smerzi, cond-mat/0403178

[13] M. Rigol and A. Muramatsu, cond-mat/0311444

[14] C. Hooley and J. Quintanilla, cond-mat/0312079

[15] The $\mathrm{x}$-axis in Fig.20 is not directly the energy in the potential because our trap bottom exhibits long term drifts. As the different data points were taken in time order a linear drift leads to a scaling of the energy axis which can be compensated by leaving the temperature in the fitting procedure as a free parameter.

[16] The asymmetry in the population of the two clouds rises from a small horizontal tilt of our magnetic trap with respect to gravity. As a consequence, the potential minimum does not coincide with the magnetic field minimum.

[17] We cannot directly calculate $E_{\text {up }}$ from the radio frequency because the bottom of our magnetic trapping potential is drifting with time and therefore $\nu_{0}$ is unknown, but we can take the position of the cloud as a reference for the energy.

[18] For this gradient, the required lattice height for a localization within one lattice site and a simultaneous suppression of Zener tunnelling is $s>10$. 